\title{
Generating 3D volumetric meshes of internal and external fruit structure
}

\author{
Mikolaj Cieslak ${ }^{1,2}$, Frédéric Boudon ${ }^{2}$, Samir Kenouche $^{3}$, Michel Zanca ${ }^{3}$, Christophe \\ Goze-Bac $^{3}$, Michel Génard ${ }^{1}$, Christophe Godin ${ }^{2}$ and Nadia Bertin ${ }^{1}$

$\begin{array}{lll}{ }^{1} \text { INRA, UR } 1115 \text { Plantes et } & { }^{2} \text { CIRAD/INRIA/INRA, } & { }^{3} \text { UMR 5221 Charles } \\ \text { Systèmes de Culture } & \text { Virtual Plants INRIA Team, } & \text { Coulomb Laboratory, } \\ \text { Horticoles, Domaine St Paul, } & \text { UMR AGAP, Avenue } & \text { Université Montpellier 2, } \\ \text { Site Agroparc, F-84914 } & \text { Agropolis, TA 108/02, } & 34090 \text { Montpellier, France } \\ \text { Avignon Cedex 9, France } & \begin{array}{l}\text { 34398 Montpellier Cedex 5, } \\ \text { France }\end{array}\end{array}$

Keywords: Solanum lycopersicum, 3D fruit modelling, fruit quality, functional-structural plant models

\begin{abstract}
Two essential functions in determining fruit quality are the transport and accumulation of water and dry matter to various fruit tissues. Since water and carbon are delivered to fruit tissues through a complex vasculature system, the internal fruit structure and pattern of vasculature may have a significant impact on their distribution within the fruit. The aim of this work is to provide methods for generating fruit structure that can be integrated with models of fruit function and used to investigate such effects. To this end, we have developed a modelling pipeline in the OpenAlea platform that involves two steps: (1) generating a 3D volumetric mesh representation of the entire fruit, and (2) generating a complex network of vasculature that is embedded within this mesh. To create the 3D volumetric mesh, we use reconstruction algorithms from the $3 \mathrm{D}$ mesh generation package of the Computational Geometry Algorithms Library. To generate the pattern of vasculature within this volumetric mesh, we use an algorithmic approach from PlantScan3D software that was designed to reconstruct tree architecture from laser scanner data. We have applied our modelling pipeline to generate the internal and external geometry of a cherry tomato fruit using Magnetic Resonance Imaging data as input. These kinds of applications of our pipeline demonstrate its ability to create species-specific models of fruit structure with relatively low effort. In another work, the volumetric meshes will be combined with models of function to form integrative computational fruit models, which will help to investigate the effects of fruit structure on quality.
\end{abstract}

\section{INTRODUCTION}

Two essential functions in determining fruit quality (size, shape, composition and texture) are the transport and accumulation of water and dry matter to various fruit tissues. Most current fruit models focus on investigating these particular functions by integrating knowledge on the physiological processes governing fruit growth without considering the structure of the fruit (Génard et al., 2007). However, since water and carbon are delivered to fruit tissues through a complex vasculature system, the internal fruit structure and pattern of vasculature may have a significant impact on the distribution 
of water and dry matter within the fruit. For example, in tomato fruit (Solanum lycopersicum) vascular patterns are known to play a role in causing the calciumdeficiency disorder blossom end rot (BER), which is associated with reduced calcium transport to the apical end of the fruit via xylem vessels (Ho and White, 2005). In addition, De Freitas et al. (2011) have shown that treating tomato plants with abscisic acid prevents BER, by, among other things, increasing the number of vascular bundles in early stages of fruit growth and producing a more regular distribution of vasculature in the placenta and pericarp tissues. Although such mechanisms can be studied using processbased models, as was done by Bar-Tal et al. (1999) for pepper fruit, these models cannot account for the effects of fruit size and shape on causing this disorder and the large gradient in calcium concentration within the fruit. Therefore, the aim of this work is to provide methods for generating fruit structure that can be integrated with models of fruit function. Such comprehensive functional-structural fruit models can then be used to study the effects of fruit structure on growth and quality.

The currently available techniques for generating 3D fruit geometry mainly focus on representing the fruit's external shape and do not offer detailed representations of the internal structure. The simplest methods represent the shape of the fruit using equationbased techniques, such as spherical equations (Saudreau et al., 2007) or ellipsoid parametric equations (Ling et al., 2007). The most common methods for generating the external 3D shape are based on reconstructions from images, for example, using contours extracted from several photographs of the whole fruit (Jancsok et al., 2001) or of slices of the fruit (Mebatsion et al., 2011). The latter method has also been applied to generate some internal compartments in the fruit by manually tracing the shape of these compartments in photographs of fruit slices (Takayama et al., 2010). All of these methods, however, represent the geometry of the fruit using 2D surfaces (polyhedra) and lack a volumetric representation, which limits their use for modelling fruit function because there is no topological information that relates the various fruit tissues. For that reason, Cieslak et al. (2012) developed a modelling pipeline that generates an internal volumetric representation of fruit. This work continues that effort by additionally generating a 3D representation of the fruit's vasculature within the volumetric mesh.

\section{MATERIALS AND METHODS}

To generate a 3D representation of internal fruit tissues and external fruit shape, we developed a modelling pipeline that includes the following steps: (1) creation of a volumetric mesh of both internal and external fruit structure, and (2) algorithmic generation of the pattern of vasculature within the fruit. We implemented this pipeline using the component-based plant modelling platform OpenAlea (Pradal et al., 2008), where both steps in the pipeline are broken-down into minimal components that are assembled together into one comprehensive model. A major advantage of using this platform is that the individual components of the pipeline can be reused or extended by others.

\section{Generating 3D volumetric mesh of fruit structure}

A first version of this procedure was presented by Cieslak et al. (2012), so here we only give a general overview. In the pipeline, a 3D volumetric mesh can be created from two types of input: (1) an external polyhedral surface or (2) 3D data from Magnetic Resonance Imaging (MRI). The MRI data is preferable because it is non-destructive and captures the internal and external structure of the fruit. In both cases, the 3D mesh 
generation package (Alliez et al., 2011) of the Computational Geometry Algorithms Library, CGAL, is used to construct the volumetric mesh. The pipeline is not yet fully automatic because segmentation of the image slices into tissue types is performed by using slice-by-slice manual tracing with ITK-SNAP software (Yushkevich et al., 2006).

\section{Generating a network of vasculature embedded within a 3D mesh}

While image-based methods can be used for generating volumetric representations of fruit tissues, it is difficult to use the same methods for generating the vasculature because of the small size of vascular bundles relative to other tissues (which also makes quantitative validation challenging). Thus, we must rely on an algorithmic approach that generates the vascular geometry based on our current knowledge of the biological processes behind vascular pattern formation. Such an approach has already been proposed for synthesizing leaf venation patterns (Runions et al., 2005) and extended to modelling 3D branching structures (Runions et al., 2007). The inspiration behind this approach is to capture the competition for resources by veins or branches growing within a defined space, which is why it is called the Space Colonization Algorithm (SCA). In the case of leaves, the veins are competing for space and are directed by discrete sources of auxin, while, in the case of trees, the buds and branches are competing for space and light. Since the control of vascular development in fruit is not completely known (De Freitas et al., 2011), in this work, the SCA is used to generate the pattern of vasculature within the fruit by simulating the competition for space. To this end, we use the PlantScan3D software (Preuksakarn et al., 2010) from OpenAlea, which was originally designed to reconstruct tree architecture from laser scanner data by means of the SCA, to generate patterns of fruit vasculature.

The PlantScan3D software accepts as input a set of points distributed in 3D space. These points serve as attractors for the growing vascular network and signify the availability of space within the fruit. To place the attraction points, we generate $N$ uniformly distributed random points within the pericarp and columella tissue of the 3D volumetric mesh representing the fruit (Figure 1A). Given these points, we use the SCA implemented in PlantScan3D to generate a multi-scale tree graph (MTG) (Godin and Caraglio, 1998), which represents the topological structure of the vascular network in the fruit. In general, an MTG defines multiscale aspects of plant topological structures, but, for our purposes, only one scale is necessary. The nodes of the graph represent small segments of vasculature and adjacent nodes are connected by edges in the graph (Figure 1B). PlantScan3D generates the MTG by iteratively adding new nodes into the graph as it fills in the space defined by the 3D mesh. Initially, the first node (the root node) is defined by the top most points in the mesh (where the fruit pedicel is) and a new node is added into the graph with each step of the algorithm. The addition of a new node at each existing node of the graph is influenced by several of the nearest attraction points, and, therefore, new nodes extend the graph in the direction of nearby attraction points. The set of attraction points that influences each node of the graph is determined by a conical volume of perception, which allows the node to sense its surrounding space. This volume is in the shape of a cone, where its height corresponds to the node's perception distance and its opening angle corresponds to the node's perception angle. These parameters are user controlled and determine the neighbourhood of attractor points that influence the addition of a new node. The growth direction is computed as the sum of normalized vectors directed towards each of the nearby attractor points. Once a new node is added to the graph, the attractor points in its neighbourhood are removed, and another iteration of 
the algorithm is performed until there are no attractor points remaining that influence existing nodes.

To set the radii of the vessels, we apply Murray's law that proposes a relation between the radius of vessels before and after a branching point (Runions et al., 2005). This is similar to the pipe model theory from trees, which relates the cross-sectional area of a parent branch to the cross-sectional areas of its child branches (Shinozaki et al., 1964). Hence, the radius of each vessel is computed according to the topological structure of the fruit's vasculature, which is stored in an MTG. Computation begins at the tips of the vasculature (the end nodes of the MTG), assigning the same initial radius to all of these vessels, and proceeds basipetally to the pedicel end of the vasculature (root node of the MTG), assigning the radius of a vessel according to the vessels it is supporting. For example, the radius $r$ of a vessel supporting two branching vessels with radii $r_{1}$ and $r_{2}$ is computed as $r^{n}=r_{1}^{n}+r_{2}^{n}$, where $n$ is a parameter that can be fitted to data (and is usually within the range from 2 to 3 ).

\section{RESULTS AND DISCUSSION}

To illustrate our modelling pipeline, we created a 3D volumetric representation of a cherry tomato fruit using MRI data, and generated the pattern of vasculature using the PlantScan3D software. The volumetric mesh is the same as the one previously generated by Cieslak et al. (2012) so it is not shown here, but Figure 1C shows the resulting pattern of vasculature.

The volumetric mesh consists of 8926 tetrahedra, with each tetrahedron labelled according to the tissue it represents (pericarp or columella). For example, all tetrahedra forming part of the pericarp are given a label of 1 and those forming part of the columella are given a label of 2 . To match the average initial volume of a cherry tomato at 10 days after anthesis (at about the start of the cell elongation phase), the entire mesh is scaled to a volume of $7 \mathrm{~cm}^{3}$. Next, a set of uniformly distributed random points serves as the attractor points in generating the pattern of vasculature (Figure 1A). For the tetrahedra forming the pericarp, 1836 points are generated with one random point per tetrahedron. For the columella, 4756 points are generated with three random points per tetrahedron. The number of random points per tetrahedron is chosen on the basis of the density of vascular vessels in the fruit tissue. A multi-scale tree graph is obtained (Figure 1B) by using these points as input into the PlantScan3D software and setting the parameters in the construction procedure as follows: the conical volume of perception is set to have an opening angle of 180 degrees and a perception distance of $0.1 \mathrm{~cm}$. To set the radii of the vessels, Murray's law is applied according to the procedure outlined in the Methods section, with $n=2$ and the initial radius of the vessels at the tips set to $0.01 \mathrm{~cm}^{2}$.

Figure 2 shows the density and mean radius of vascular vessels in the pericarp and columella tissues generated according to the parameters above. These values are calculated over four even horizontal layers of the fruit from the pedicel end to the apical end, i.e., the mesh is divided into four horizontal layers from top to bottom. The results show that the density of vessels in the pericarp and columella decreases from pedicel to apical ends, which is in general agreement with observations of the real fruit (De Freitas et al., 2011) and in accordance with the hypothesised cause of BER. Furthermore, the results show a decreasing average radius of vessels from the pedicel to apical ends of the fruit in both tissues, which is a result of the application of Murray's law. The interpretation of these results, however, is difficult because measurements of vascular vessels in fruit is challenging. A much more thorough analysis would require fitting the 
parameters of the vasculature generation algorithm and making a qualitative and quantitative comparison with biological data. Such data could come from similar measurements to those made by De Freitas et al. (2011) of the number of stained vascular vessels $\mathrm{cm}^{-2}$ in a tomato fruit at several time periods after pollination.

\section{CONCLUSIONS}

The main feature of our modelling pipeline is the generation of 3D fruit structure, including the external shape and internal tissues. In this paper, we have extended the volumetric mesh generation methods proposed by Cieslak et al. (2012) with generation of fruit vasculature. This semi-automated generation of fruit geometry from images or MRI data coupled with the algorithm generation of vasculature enables creation of speciesspecific based models of fruit structure with relatively low effort. Such an approach that integrates various steps in generating a $3 \mathrm{D}$ volumetric mesh is possible because our modelling pipeline is implemented within the OpenAlea framework, which also simplifies its reusability. Currently, the only step that requires extensive user interaction is the segmentation of MRI data into different tissue types by manual slice-by-slice tracing. In the future, this step could be automated using segmentation routines from the MARSALT package (Fernandez et al., 2010) of OpenAlea, which was designed for similar processing of data on the shoot apical meristem.

The present study concentrates on presenting only the geometric aspects of a comprehensive fruit model. In another work, volumetric meshes are being combined with models of function to form integrative computational models of the fruit (functionalstructural models). The result will be a dynamic system that gives us the ability to model fruit growth driven by exogenous factors like temperature and humidity. With this type of functional-structural model, it would be possible to investigate the role of asymmetric vascular structure in determining the distribution of water and dry matter in the fruit, and to show the dependence of this distribution on vascular geometry.

\section{ACKNOWLEDGEMENTS}

We thank Chakkrit Preuksakarn for advice on using the PlantScan3D software, and Christophe Pradal for advice in development of our pipeline using the OpenAlea framework. This project is made possible through the financial support of Agropolis Fondation.

\section{Literature Cited}

Alliez, P., Rineau, L., Tayeb, S., Tournois, J., and Yvinec, M., 2011. 3D Mesh Generation, In CGAL User and Reference Manual. CGAL Editorial Board.

Bar-Tal, A., Keinan, M., Fishman, S., Aloni, B., Oserovitz, Y., and Génard, M. 1999. Simulation of environmental effects on Ca content in pepper fruit. Acta Horticulturae 507:253-262.

Cieslak, M., Génard, M., Kenouche, S., Goze-Bac, C., Godin, C., and Bertin, N. 2012. Towards a 3D virtual fruit model integrating fruit architecture and physiology. Acta Horticulturae:To appear.

de Freitas, S.T., Shackel, K.A., and Mitcham, E.J. 2011. Abscisic acid triggers wholeplant and fruit-specific mechanisms to increase fruit calcium uptake and prevent blossom end rot development in tomato fruit. J Exp Bot 62:2645-2656. 
Fernandez, R., Das, P., Mirabet, V., Moscardi, E., Traas, J., Verdeil, J.L., Malandain, G., and Godin, C. 2010. Imaging plant growth in 4D: robust tissue reconstruction and lineaging at cell resolution. Nat Methods 7:547-U594.

Génard, M., Bertin, N., Borel, C., Bussières, P., Gautier, H., Habib, R., Léchaudel, M., Lecomte, A., Lescourret, F., Lobit, P., and Quilot, B. 2007. Towards a virtual fruit focusing on quality: modelling features and potential uses. J Exp Bot 58:917-928.

Godin, C. and Caraglio, Y. 1998. A multiscale model of plant topological structures. Journal of Theoretical Biology 191:1-46.

Ho, L.C. and White, P.J. 2005. A cellular hypothesis for the induction of blossom-end rot in tomato fruit. Ann Bot-London 95:571-581.

Jancsok, P.T., Clijmans, L., Nicolai, B.M., and De Baerdemaeker, J. 2001. Investigation of the effect of shape on the acoustic response of 'conference' pears by finite element modelling. Postharvest Biol Tec 23:1-12.

Ling, L., Hongzhen, X., Wenlin, S., and Gelin, L. 2007. Research on visualisation of fruits based on deformation. New Zeal J Agr Res 50:593-600.

Mebatsion, H.K., Boudon, F., Godin, C., Pradal, C., Genard, M., Goz-Bac, C., and Bertin, N. 2011. A novel profile based model for virtual representation of quasisymmetric plant organs. Comput. Electron. Agric. 75:113-124.

Pradal, C., Dufour-Kowalski, S., Boudon, F., Fournier, C., and Godin, C. 2008. OpenAlea: a visual programming and component-based software platform for plant modelling. Funct Plant Biol 35:751-760.

Preuksakarn, C., Boudon, F., Ferraro, P., Durand, J.-B., Nikinmaa, E., and Godin, C., 2010. Reconstructing plant architecture from 3D laser scanner data, p. 16-18. In: de Jong, T. and Da Silva, D. (eds.), 6th International Workshop on FunctionalStructural Plant Models. University of California, Davis, USA.

Runions, A., Fuhrer, M., Lane, B., Federl, P., Rolland-Lagan, A.G., and Prusinkiewicz, P. 2005. Modeling and visualization of leaf venation patterns. Acm T Graphic 24:702-711.

Runions, A., Lane, B., and Prusinkiewicz, P. 2007. Modeling trees with a space colonization algorithm. Proceedings of the 2007 Eurographics Workshop on Natural Phenomena 63-70.

Saudreau, M., Sinoquet, H., Santin, O., Marquier, A., Adam, B., Longuenesse, J.J., Guilioni, L., and Chelle, M. 2007. A 3D model for simulating the spatial and temporal distribution of temperature within ellipsoidal fruit. Agr Forest Meteorol 147:1-15.

Shinozaki, K., Yoda, K., Hozumi, K., and Kira, T. 1964. A quantitative analysis of plant form---the pipe model theory. I. Basic analyses. Japanese Journal of Ecology 14:97-105.

Takayama, K., Sorkine, O., Nealen, A., and Igarashi, T. 2010. Volumetric Modeling with Diffusion Surfaces, New York, NY, USA 29).

Yushkevich, P.A., Piven, J., Hazlett, H.C., Smith, R.G., Ho, S., Gee, J.C., and Gerig, G. 2006. User-guided 3D active contour segmentation of anatomical structures: Significantly improved efficiency and reliability. Neuroimage 31:1116-1128. 


\section{Figures}
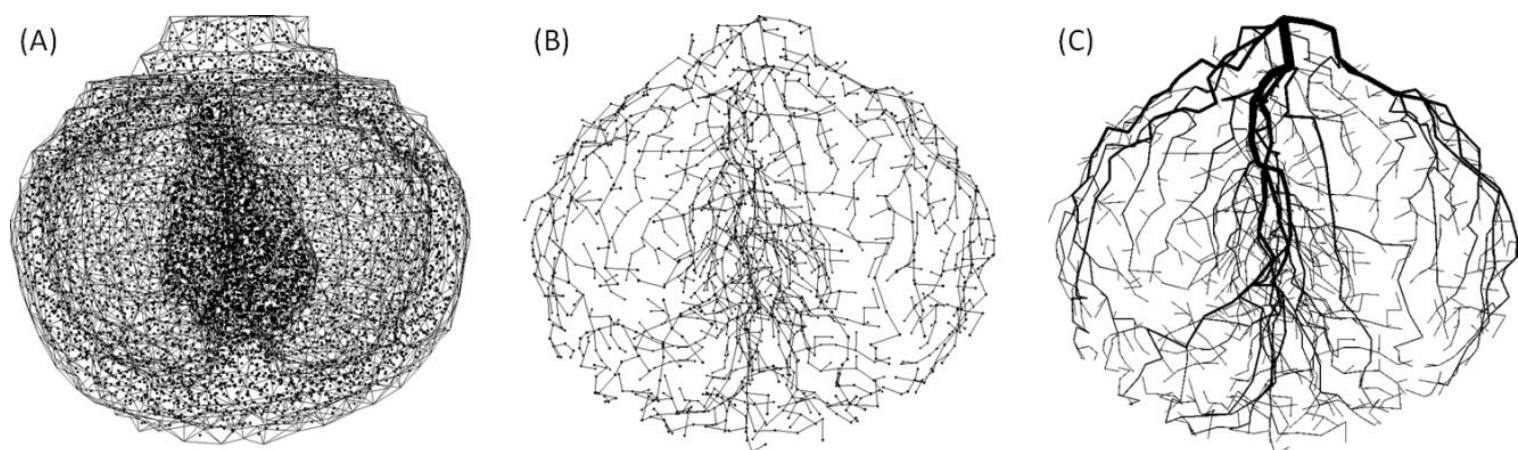

Fig. 1. Generation of vascular geometry within a 3D volumetric mesh: (A) the input to the PlantScan3D software is a set of attraction points specified uniformly within the pericarp and columella tissues, (B) the output of the software is a multi-scale tree graph (MTG) representing the topological structure of the vasculature, and $(C)$ the final result is a $3 \mathrm{D}$ representation of vascular geometry, which is obtained after processing the MTG and setting the radii of vessels according to Murray's law. The middle image (B) shows the vascular geometry before radii of vessels are computed, whereas the right image (C) shows the geometry after the radii are computed.
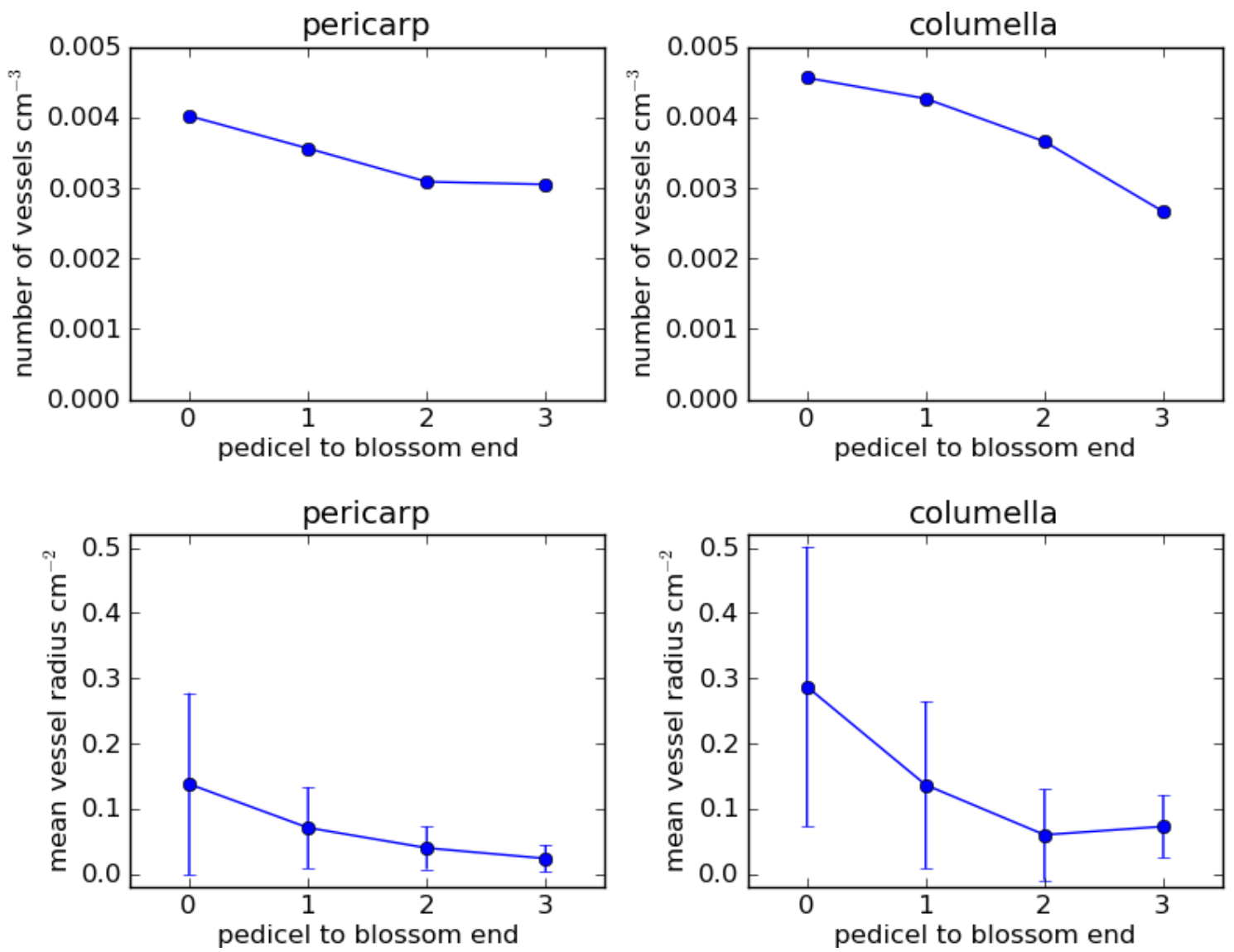
Fig. 2. Density and mean radius of the vasculature in the pericarp and columella tissues computed from a 3D volumetric mesh representing a cherry tomato fruit. The mesh is divided into four even horizontal layers and these values are computed within each layer. The density of vessels is computed as the total number of vessels in a layer divided by the total volume of that layer. The mean radius is found over all the vessels within each layer, with bars indicating the standard deviation. 